\title{
Study on characterization of Furcraea foetida new natural fiber as composite reinforcement for lightweight applications
}

\begin{abstract}
The exploration of new natural fibers in the field of polymer composites can contribute to increase the invention of natural reinforcements and expand their use in possible applications. In the present work, the physico-chemical, thermal, tensile and morphological properties of Furcraea foetida (FF) fiber are presented for the first time. Chemical analysis results shows that FF has relatively higher cellulose $(68.35 \%)$ with lower hemicelluloses $(11.46 \%)$ and lignin (12.32\%). Structural analysis of FF was conducted by Fourier transform infrared and 13C (CPMAS) nuclear magnetic resonance spectroscopy. X-ray diffraction (XRD) analysis evidenced that FF has crystallinity index of $52.6 \%$ with crystalline size of $28.36 \mathrm{nmThe}$ surface morphology of FF was investigated by scanning electron microscopy (SEM), energy dispersive $\mathrm{X}$-ray micro analyzer (EDX) and atomic force microscopy (AFM). The thermogravimetric analysis (TGA) reveals thermal constancy of the fiber upto $320.5^{\circ} \mathrm{C}$ with the kinetic activation energy of $66.64 \mathrm{~kJ} / \mathrm{mol}$, which can be used as reinforcements in thermoplastic green composite whose working temperatures is below $300{ }^{\circ} \mathrm{C}$. The FF results were compared with those of other natural fibers, and indicated as a suitable alternative source for composite manufacture.
\end{abstract}

Keyword: Furcraea foetida fiber; Chemical analysis; X-ray diffraction; Fourier transform infrared; NMR spectroscopy; Thermal analysis; Tensile test; Morphological properties 